\title{
A Collaborator's Reputation Can Bias Decisions and Anxiety under Uncertainty
}

\author{
Song Qi, ${ }^{1,2 \star}$ Owen Footer, ${ }^{2 \star} \mathbb{C}^{C}$ Colin F. Camerer, ${ }^{1}$ and ${ }^{-D e a n}$ Mobbs ${ }^{1,2}$ \\ ${ }^{1}$ California Institute of Technology, Pasadena, California 91125, and ${ }^{2}$ Columbia University, New York, New York 10027
}

Informational social influence theory posits that under conditions of uncertainty, we are inclined to look to others for advice. This leaves us remarkably vulnerable to being influenced by others' opinions or advice. Rational agents, however, do not blindly seek and act on arbitrary information, but often consider the quality of its source before committing to a course of action. Here, we ask the question of whether a collaborator's reputation can increase their social influence and, in turn, bias perception and anxiety under changing levels of uncertainty. Human male and female participants were asked to provide estimations of dot direction using the random dot motion (RDM) perceptual discrimination task and were paired with transient collaborators of high or low reputation whom provided their own estimations. The RDM varied in degrees of uncertainty and joint performance accuracy was linked to risk of an electric shock. Despite providing identical information, we show that collaborating with a high reputation compared with a low reputation partner, led to significantly more conformity during the RDM task for uncertain perceptual decisions. Consequently, high reputation partners decreased the subjects' anxiety during the anticipatory shock periods. fMRI data showed that parametric changes in conformity resulted in increased activity in the ventromedial PFC, whereas dissent was associated with increased in activity in the dorsal anterior cingulate cortex (dACC). Furthermore, the dACC and insula, regions involved in anticipatory pain, were significantly more active when collaborating with a low reputation partner. These results suggest that information about reputation can influence both cognitive and affective processes and in turn alter the neural circuits that underlie decision-making and emotion.

Key words: anxiety; conformity; decision; fMRI; pain; reputation

\section{Significance Statement}

Humans look to others for advice when making decisions under uncertainty. Rational agents, however, do not blindly seek information, but often consider the quality of its source before committing to a course of action. Here, we ask the question of whether a collaborators' reputation can increase social influence and in turn bias perception and anxiety in the context of perceptual uncertainty. We show that when subjects are partnered with collaborators with a high reputation, this leads to increased conformity during uncertain perceptual decision-making and reduces anxiety when joint performance accuracy leads to an electric shock. Furthermore, our results show that information about reputation alters the neural circuits that underlie decision-making and emotion.

\section{Introduction}

According to psychological theory, when we are uncertain about what actions to take, we often look to others to guide our deci-

\footnotetext{
Received Aug. 17, 2017; revised Dec. 4, 2017; accepted Jan. 13, 2018

Author contributions: D.M. and O.F. designed research; 0.F. performed research; S.Q. and 0.F. analyzed data; D.M., C.C., S.Q., and O.F. wrote the paper.

This work was supported by the National Institute of Mental Health (NIMH) Grant 2P50MH094258 (D.M. and C.F.C.), and startup funds from Columbia University. The content is solely the responsibility of the authors and does not necessarily represent the official views of the funders.

The authors declare no competing financial interests.

*S.Q. and O.F. contributed equally to this work.

Correspondence should be addressed to Dr. Dean Mobbs, Humanities and Social Sciences, and Computation and Neural Systems Program, 319 Baxter Hall, California Institute of Technology (Caltech), Pasadena, CA 91125. E-mail: dmobbs@caltech.edu.
}

sions (Cialdini, 1993; Wooten and Reed, 1998). In turn, this leaves us remarkably vulnerable to being influenced by others' opinions or advice. This is common in daily life as important decisions are often based on limited information, and under such circumstances, we often look to the advice of others whom we have a high regard for, namely those with a good reputation. Supporting the conjecture that reputation can have a potent influence on social behavior is research showing that we are highly sensitive to the reputation and the competence of others when making decisions (Todorov et al., 2005; Boorman et al., 2013; Tedeschi et al., 2015). Further, classic social psychological exper- 
A

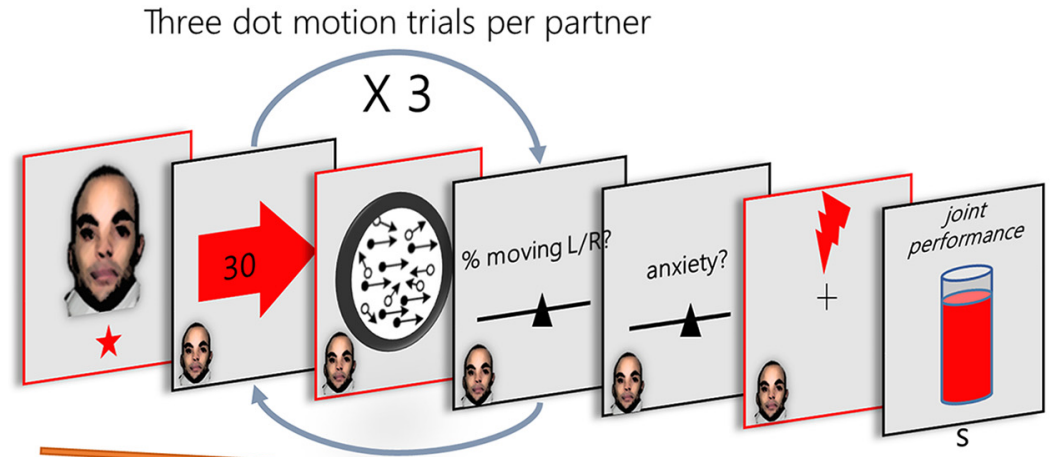

$3 \mathrm{~s}$

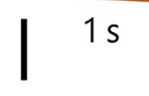

$3 \mathrm{~s}$

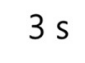

$3 \mathrm{~s}$

$3 \mathrm{~s}$

4-6s
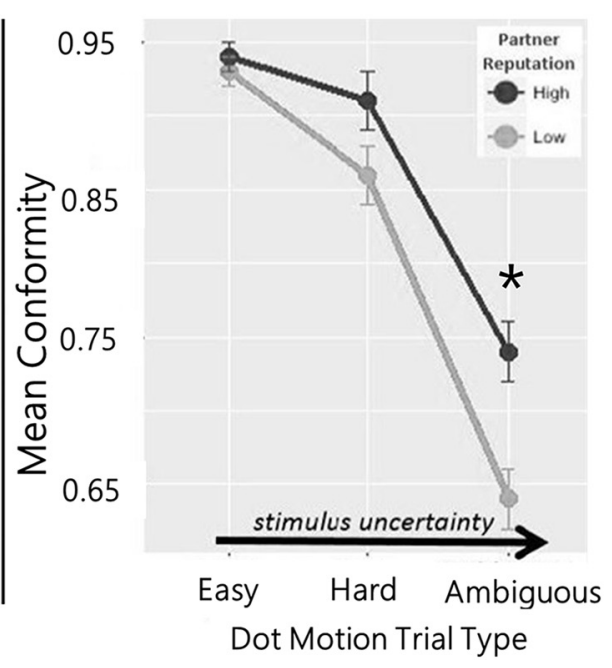

Figure 1. Task paradigm and behavioral results. $A$, Experimental steps: the subject was first shown a picture of the partner's face and reputation $\left(1 \mathrm{star}=\operatorname{Rep}_{\text {Low }}\right.$ or 5 stars $=$ Rep $_{\text {High }}$ ). Next, the subject was shown two screens: (1) an arrow indicating the partner's guess about the direction of the dots (arrow screen) and (2) their guess about the coherence percentage (30\% in the example). The subject then saw a screen showing the dot movement and was asked to guess the coherence percentage (using a slider scale). The RDM discrimination estimation was repeated three times. Next, subjects reported how anxious they feel at the prospect of receive a shock during the $4-6 \mathrm{~s}$ anticipation screen. The likelihood of receiving a shock was based on the joint performance accuracy between the subject and the partner. After the shock anticipation screen, they either received a shock or not. Then they saw a screen displaying information about the joint performance of themselves and the partner. The red-bordered boxes are the analyzed events. $\boldsymbol{B}$, Left, Conformity was higher for high-reputation partners. Right, Mean conformity differed across hard and ambiguous (uncertain), but not easy conditions. ${ }^{*} p<.001$.

iments demonstrate subjects' susceptibility to conforming, that is changing one's behavior to match opinions and actions of others (Sherif, 1935; Schachter, 1951; Asch, 1956; Cialdini and Goldstein, 2004) is especially evident when the influencer is high in authority or reputation (Milgram, 1964). Despite this large body of research, no studies have yet attempted to understand how the reputation of others can modulate social influences on perceptual discrimination.

The information we garner from reputable others and how it influences our decisions become critical when it relates to decision outcomes that result in physical harm. For example, cagereared monkeys who are not afraid of snakes instantly exhibit fear after they observe another conspecific exhibit fear of snakes (Mineka and Cook, 1986). It is known that, in general, the mere presence of a conspecific can ameliorate an individual's response to a stressor, a phenomenon called "social buffering" (Kikusui et al., 2006). The identity of the present conspecific can also change the strength of the buffering response. For example, holding the hand of a romantic partner during the anticipation of a possible electric shock reduced the neural pain responses (Coan et al., 2006). Others have shown that when the risk of a shock depends on the task performance of another person, the level of perceived competence extracted from facial features modulates both subjective and neural measures of anxiety (Tedeschi et al., 2015). Theories of such findings (Beckes and Coan, 2011; Krahé et al., 2013) posit that conspecifics reduce threat responses if they offer protective resources that factor into predictive computations of threat severity. Little is known, however, about what properties of conspecifics, such as wisdom, experience, competence, or reputation, modulate social buffering.

To explore the relationship between reputation, perceptual bias and emotion, we used functional MRI in conjunction with a standard random dot motion (RDM) discrimination task to test whether partners high or low in reputation would modulate perceptual decisions and anxiety of the subject. To create reputation ratings of the partners, subjects were shown the average ratings of each partner made by their friends [rounded off to either 1 (low), 3 (mid), and 5 (high) reputation]. Because perception and anxiety both rely heavily on expectations to inform interpretations of ambiguous stimuli (Petrovic et al., 2005; Sterzer et al., 2008), we hypothesized that a partner's reputation would play a significant role determining the degree of social influence on perception and anxiety. In the task, healthy subjects were provided with RDM estimates by transient collaborators of high or low reputation. The subject's goal was to make RDM estimates based on their own judgements and where needed, incorporate the RDM estimates of the transient collaborator (Fig. 1). The likelihood of an electric shock at the end of each block is based ostensibly on combined task

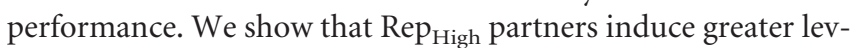
els of social influence (i.e., conformity) during the RDM discrimination task, especially for the uncertain conditions and downregulate subjective and neural markers of anxiety.

\section{Materials and Methods}

Participants. Twenty-five individuals recruited from the Columbia University community completed all parts of the experiment. Data from three participants were removed due to excessive head motion during scanning (1) or technical issues (shocks not working; 2), and all remaining analyses are based on the remaining 22 individuals ( 10 females, mean age $=25.6 \pm 4.6$, range 19-35). All participants were right-handed, had normal or corrected-to-normal vision, had no history of neurological or psychiatric illness, and gave written informed consent for participation. The study was approved by the Institutional Review Board of Columbia University. 
Experimental paradigm. The experiment consisted of a 42 min session consisting of 48 blocks. In each block (Fig. 1), the participant was paired up with a new "partner", or someone who ostensibly played the same task on a previous visit to the psychology department. At the beginning of each block, the participant was presented with a picture of that partner along with a star rating below. The pictured partners had neutral facial expressions, were edited to an identical size and brightness, and were unfamiliar to subjects (as confirmed in a debriefing questionnaire). Confounds such as attractiveness, trustworthiness, gender, race, and the like were minimized by counterbalancing the faces across subjects.

Implicit competence was controlled for by using ratings from independent samples of raters from the same subject population (from multiple samples, total $N=91$ ). Raters judged each partner's competence based solely on the partner's picture (Tedeschi et al., 2015). Ratings were trifurcated into 1, 3, or 5 stars (with more stars indicating better judged competence). Participants were instructed that the ratings were an average from the partners' friends' judgments of their general skill at perceptual, cognitive, or "mind training" games. This was made more realistic by asking the subject to give the names of friends who could rate them on their perceptual and cognitive abilities and by taking a photograph for future participants in the experiment. Before starting the task each participant was randomly assigned to one of two counterbalanced sets of pairings between partner pictures and reputation ratings. After viewing the partner's picture and star rating for $3 \mathrm{~s}$ at the start of the block, the star rating disappeared and a small thumbnail picture of the partner remained in a screen corner. There were also four blocks ( 2 in each session) without a partner; in those blocks the partner's picture and rating were replaced with "no partner" text.

After the $3 \mathrm{~s}$ partner and rating presentation, a 2-4 s jittered ISI occurred before the perception task began. The goal for participants was to make accurate perceptual judgments ( 3 per block) of the direction and level of motion coherence in the RDM discrimination task. The RDM discrimination task is a standard psychophysical stimulus used to study motion perception (Newsome et al., 1989; Raymond, 2000). In the RDM, most dots on the screen move in random directions but a portion of the total dots move coherently together either left or right. Coherence levels ranged from 0 to $40 \%$, indicating the percentage of all dots on the screen that are moving together. Participants must judge whether the coherent dots are moving left or right (binary judgment), as well as the numerical level of coherence from 0 to $50 \%$ in steps of $5 \%$. As with the rest of the task, dot stimuli were presented using PsychoPy software based in Python, using the built-in Dots Component function with the following parameters: 100 total dots on the screen, 10 pixels dot size, 0.01 U/frame speed, 1000 frame dot lifetime, and noise dots follow constant direction. Each dot stimulus was presented for $3 \mathrm{~s}$, after which the participant had $3 \mathrm{~s}$ to input their judgment by toggling left or right on a scale that started in the middle at $0 \%$ coherence. A $2-4 \mathrm{~s}$ jittered ISI followed each rating.

In the blocks that included a partner, participants saw their partner's judgment for $1 \mathrm{~s}$ before each dot stimulus in the form of an arrow pointing left or right (indicating the partner's binary judgment) and a number inside the arrow (indicating the partner's continuous coherence judgment). In blocks without a partner, a blank screen replaced the arrow for the $1 \mathrm{~s}$ before the dot stimulus. Each block consisted of three dot motion trials, including one "easy" trial (30-40\% coherence), one "hard" trial ( $10-20 \%$ coherence), and one "ambiguous" trial ( $0 \%$ coherence). There was no difference in task difficulty or the quality of partner judgments by partner reputation. By definition, however, there were differences in the quality of partner judgments by partner performance levels, such that the total error of the judgments provided by low performing partners (70$80 \%$ total error) was higher than that of high performing partners (10$20 \%$ total error). Subjects could select "no direction" by selecting $0 \%$ coherence on the visual analog scale. Participants were not explicitly instructed about the different levels of task difficulty, or the possibility that some trials would not have a true direction left/right. They were also not instructed on how they should use the information provided by the partner. Accuracy was calculated from the absolute distance between the subject's indicated coherence and the actual coherence of the dots.
Near the end of each block was an anticipation period (4-6s, jittered) during which participants were instructed that they may receive an electric shock to the left wrist at any point. Before each anticipation period, participants were given $3 \mathrm{~s}$ to rate their level of anxiety on a 7-point Likert scale. Participants were told the shocks were probabilistically based on a lower combined performance of both themselves and their partner, such that worse combined performance was associated with a larger probability of shock. For blocks without a partner, participants were instructed the probability of getting a shock was based solely on their own performance. In reality, all participants received the same number of shocks (5) and those shocks were paired with the same partners within each of the counterbalanced sets of partners. The shock stimuli were delivered using a Biopac MP150 with an STM100C module (Biopac Systems). Attached to the STM100C was a $200 \mathrm{~V}$ maximum stimulus isolation unit (STMISOC, Biopac Systems). Shocks were administered via pre-gelled radio translucent electrodes on the underside of the participant's left wrist and attached to the STMISOC with shielded leads. The shocks were calibrated for each participant before the scan with a procedure that allowed the participant to select a shock level that was uncomfortable but not too painful, with an upper limit of $100 \mathrm{~V}$.

After the anticipation period and a 2-4 s jittered ISI there was a $3 \mathrm{~s}$ feedback period. During this time, participants were shown a visual metric (a partially full cylinder) ostensibly indicating the combined performance of the partner and participant in that block. In reality this feedback bar only reflected the partner's performance indexed by the amount of total error in his/her three judgments. Importantly, the partner's performance was orthogonal to the reputation rating, and both were orthogonal to the partner's implicit competence, gender, and race.

fMRI data acquisition. All fMRI data were acquired using a GE Discovery MR750 3.0 T scanner with 32-channel head coil. The imaging session consisted of two function scans, each $20 \mathrm{~min}$, as well as a high-resolution anatomical T1-weighted image ( $1 \mathrm{~mm}$ isotropic resolution) collected at the beginning of each scan session. For functional imaging, interleaved $\mathrm{T} 2{ }^{\star}$-weighted gradient-echo echoplanar imaging (EPI) sequences were used to produce 453 -mm-thick oblique axial slices $(\mathrm{TR}=2 \mathrm{~s}$, $\mathrm{TE}=25 \mathrm{~ms}$, flip angle $=77^{\circ}, \mathrm{FOV}=192 \times 192 \mathrm{~mm}$, matrix $\left.=64 \times 64\right)$.

fMRI data preprocessing. Structural images were subjected to the Unified Segmentation algorithm implemented in SPM8, yielding discrete cosine transform spatial warping coefficients used to normalize (warp) each individual's data (structural and functional) into MNI space. After discarding the first five volumes of each functional run to account for equilibrium effects, the functional data were preprocessed using the following SPM8 functions: slice-time correction, two-pass realignment to correct for head motion (rigid body registration of all frames to the averaged image after first pass), coregistration of each participant's functional mean image to the corresponding structural image, followed by applying the normalization parameters determined during segmentation to the functional images, and then using a $6 \mathrm{~mm}$ FWHM Gaussian smoothing kernel.

Statistical analysis of $f M R I$ data. Preprocessed images were subjected to a two-level general linear model using SPM8. The first (individual participant) level contained the following regressors of interest, each convolved with the canonical two-gamma hemodynamic response function: a $3 \mathrm{~s}$ boxcar function for the partner and rating presentation period, a $3 \mathrm{~s}$ boxcar function for the dot motion perception period, a 4-6s (durationjittered) boxcar function for the anticipation period, and a $3 \mathrm{~s}$ boxcar function for the feedback period. In addition, an orthogonal regressor using the mean-centered anxiety ratings parametrically modulating the anticipation period was used, as well as orthogonal regressors using mean-centered dot motion coherence and computed conformity levels parametrically modulating the motion perception period. Regressors of no interest consisted of motion parameters determined during preprocessing, their first temporal derivative, and discrete cosine transformbased temporal low-frequency drift regressors with a cutoff of $192 \mathrm{~s}$.

Beta (regression weight) maps were used to create linear contrast maps (weighted sums of betas), which were then subjected to several secondlevel, random-effects (summary statistics) one-sample $t$ tests, with the null hypothesis being that the mean over all participants is zero (0). Our model included the partner and rating presentation period $(3 \mathrm{~s})$, the 

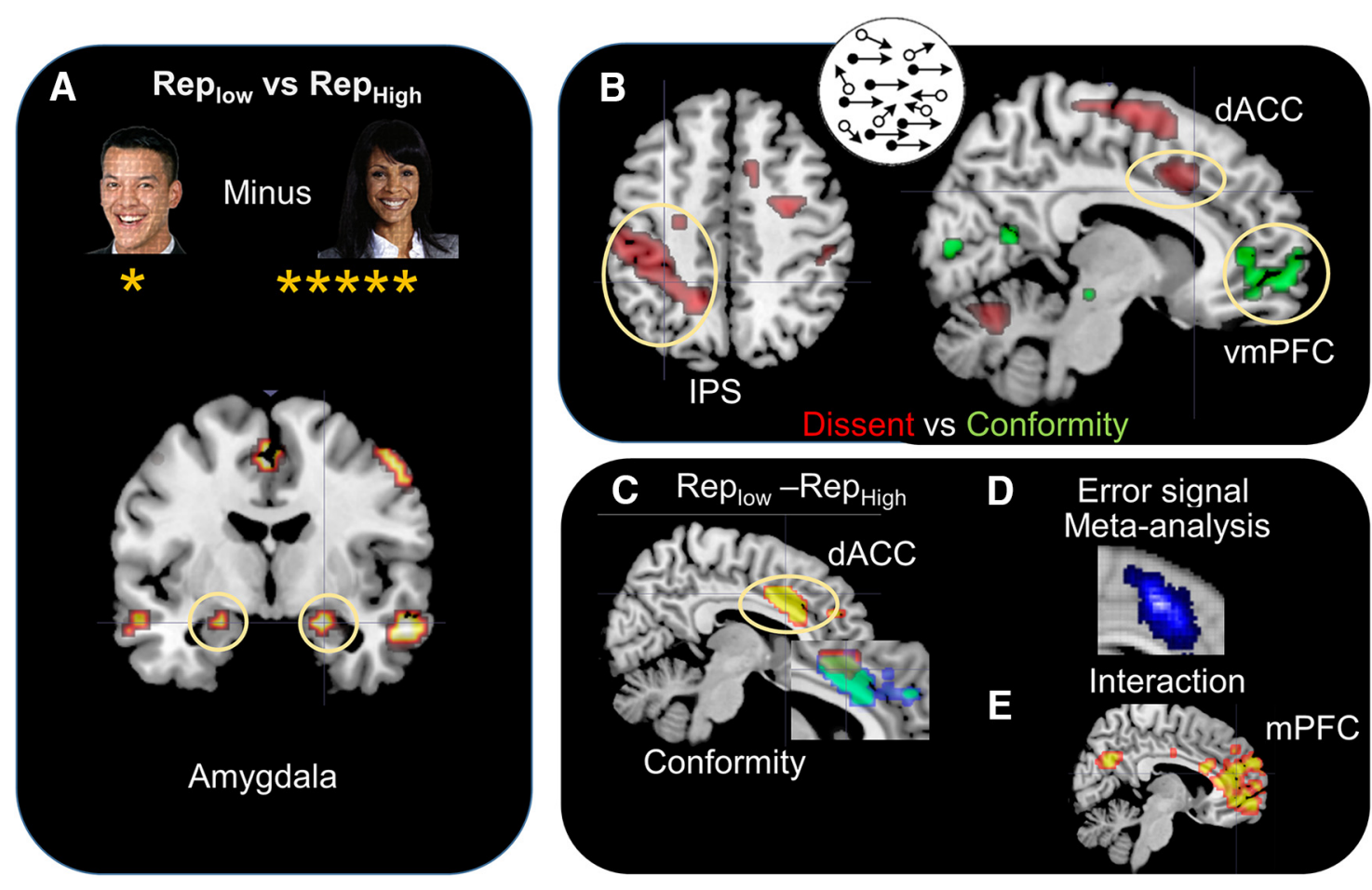

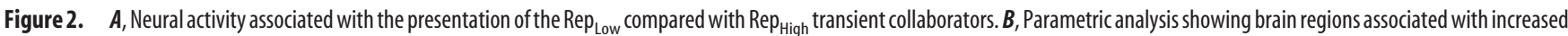
dissent and conformity during the RDM task. C, dACC activity associated with increased conformity with the Rep Low $_{\text {compared with Rep }}$ High and inset showing overlap between regions associated with dissent and Rep Low conformity. D, Neurosynth meta-analysis of 357 studies using the search term "Error" (cluster represent a forward inference); $(\boldsymbol{E})$ medial PFC activity for the $2 \times 2$ interaction between $\operatorname{Rep}_{\text {High }} / \operatorname{Rep}_{\text {Low }} \times$ Easy/Uncertain RDM conditions.

motion perception period ( 3 trials each of $3 \mathrm{~s}$ ), the anticipation period $(4-6 s)$, and the feedback period ( $3 \mathrm{~s})$. The analyses only included anticipation periods when a shock did not occur. The resulting statistical maps (one-sample $t$ tests) were thresholded at $p<0.05$, corrected for multiple comparisons, and false discovery rate (FDR)-corrected. (Genovese et al., 2002). Additionally, for results with strong a priori spatial hypotheses, a small volume correction (SVC) was applied.

Statistical analysis of behavioral data. All behavioral data were analyzed in $\mathrm{R}$. The task was a $2 \times 2$ factorial design with two main independent variables, partner reputation and partner performance. Each of these two variables had three levels, but there were fewer instances of the midlevel star ratings and performance bars since they were used mainly for psychological validity. The low and high levels of both variables were balanced and were the main conditions of interest in the analyses, therefore the task was treated as a $2 \times 2$ factorial design for analysis of the anxiety measures. Further, the "lmer" package in R was used for to test hypotheses on the repeated-measures data with linear mixed-effect models. With a binary-dependent variable, we used a generalized linear mixed model with a logit link fit by maximum likelihood ("glmerMod"), which provides $z$-statistics for hypothesis testing of the fixed effects. With a continuous dependent variable, we used a linear mixed model fit by REML ("lmerMod"), which provides $t$ statistics using a noninteger Satterthwaite approximation to degrees of freedom for the fixed effects.

\section{Results}

Partner reputation

We first analyzed the fMRI signal in response to the reputation of the partner at the time of the initial partner screen display. The amygdala (MNI-coordinates $-18,0,-24 ; p=0.020$ SVC),

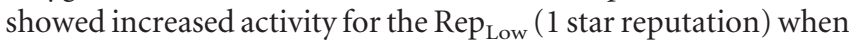
compared directly to the $\operatorname{Rep}_{\text {High }}$ (5 star reputation; Fig. $2 A$ ). Other activated regions including the precuneus, hippocampus and $\mathrm{mPFC}$ were also activated $[p<0.05$ whole-brain corrected (WBC); Table 1]. No differential activity was observed for the Rep $_{\text {High }}$ condition compared with Rep Low.
Table 1. Brain activation for contrast [low reputation $>$ high reputation (partner period)]

\begin{tabular}{|c|c|c|c|c|c|c|}
\hline \multirow[b]{2}{*}{ Brain region } & \multirow[b]{2}{*}{ Left/right } & \multirow[b]{2}{*}{ Cluster size } & \multirow[b]{2}{*}{ tscore } & \multicolumn{3}{|c|}{ Coordinates } \\
\hline & & & & $x$ & $y$ & $z$ \\
\hline \multicolumn{7}{|c|}{ Low reputation $>$ high reputation (partner) } \\
\hline Cerebellum & $\mathrm{R}$ & 1657 & 8.50 & 39 & -54 & -24 \\
\hline Precuneus & $\mathrm{L}$ & 556 & 8.32 & -3 & -54 & 15 \\
\hline $\mathrm{mPFC}$ & $\mathrm{R}$ & 253 & 7.77 & 3 & 57 & -9 \\
\hline Hippocampus & $\mathrm{L}$ & 130 & 6.37 & -18 & -6 & -18 \\
\hline Middle temporal gyrus & $\mathrm{R}$ & 61 & 6.26 & 54 & 0 & -21 \\
\hline Superior motor area & $\mathrm{L}$ & 82 & 5.44 & -3 & -6 & 51 \\
\hline Amygdala* & $\mathrm{L}$ & 14 & 3.56 & -18 & 0 & -24 \\
\hline
\end{tabular}

All $p<0.05$, corrected for multiple comparisons (FDR).

*SVC correction.

Partner reputation and RDM coherence estimates

Next we show that subjects were no more accurate in their judgments of the RDM direction when paired with Rep Low $_{\text {compared }}$ with $\mathrm{Rep}_{\text {High }}$ partner $\left(88.5 \%\right.$ vs $86 \%$ correct; $t_{(21)}=1.14, p=$ 0.13 ; one-sample $t$ test). The partner's influence on participant judgments, however, was significantly higher for Rep $\mathrm{p}_{\text {High }}$ compared with $\operatorname{Rep}_{\text {Low }}$, based on both binary measures of social influence (whether partner and participant judgments of motion direction match; $z=2.48, p=0.01$; Fig. $1 B$ ) as well as a continuous measure (the absolute difference in judgments of motion coherence; $t_{(21)}=-2.80, p=0.01$; one-sample $t$ test). There was also an interaction between task difficulty and partner reputation on the amount of social influence on perception. Task difficulty is equated to stimulus uncertainty (lower coherence corresponds to higher stimulus uncertainty and makes the task more difficult). As difficulty increased, the difference in the amount of social

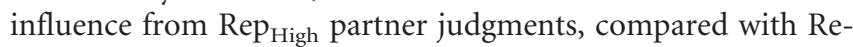


Table 2. Brain activation for parametric modulation of conformity

\begin{tabular}{lllllllr}
\hline & & & & \multicolumn{3}{c}{ Coordinates } \\
\cline { 5 - 7 } Brain region & Left/right & Cluster size & tscore & $x$ & $y$ & $z$ \\
\hline Conformity + & & & & & & \\
$\quad$ Fusiform & $\mathrm{R}$ & 34 & 5.6 & 24 & -69 & -12 \\
mPFC & $\mathrm{L}$ & 182 & 5.53 & -6 & 57 & 0 \\
Postcentral gyrus & $\mathrm{R}$ & 112 & 5.23 & 60 & -9 & 30 \\
Insula & $\mathrm{R}$ & 115 & 4.97 & 45 & 3 & 3 \\
\hline
\end{tabular}

All $p<0.05$, corrected for multiple comparisons (FDR).

Table 3. Brain activation for parametric modulation of conformity (low reputation $>$ high reputation)

\begin{tabular}{lccrrrr}
\hline & & & \multicolumn{3}{c}{ Coordinates } \\
\cline { 5 - 7 } Brain region & Left/right & Cluster size & $t$ score & $x$ & $y$ & $z$ \\
\hline Conformity + (low-high reputation) & & & & & & \\
$\quad$ Caudate & $\mathrm{R}$ & 57 & 5.36 & 13 & 7 & -9 \\
Precentral gyrus & $\mathrm{L}$ & 48 & 5.22 & -45 & -6 & 48 \\
Superior temporal gyrus & $\mathrm{R}$ & 94 & 5.11 & 58 & -29 & 15 \\
dACC & $\mathrm{R}$ & 203 & 4.97 & 9 & 23 & 27 \\
\hline
\end{tabular}

All $p<0.05$, corrected for multiple comparisons (FDR).

$\mathrm{p}_{\text {Low }}$, also increased $\left(t_{(21)}=-1.79, p=0.04\right.$; one-sample $t$ test; Fig. $1 C$ ). When the task was easy, there was no significant difference in measures of social influence between Rep High $_{\text {compared }}$

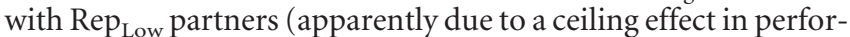
mance). When the task was hard or ambiguous (uncertain), there was significantly more conformity to $\mathrm{Rep}_{\mathrm{High}}$ compared with Rep Low partners $\left(t_{(21)}=-3.54, p<0.001\right.$; one-sample $t$ test $)$.

To investigate the neural systems underlying these differing levels of social influence between partner reputation conditions, we looked at the fMRI data during RDM task. We first examined the parametric changes associated with increasing conformity and increasing dissent from the partner's judgment (Table 2). Increasing conformity was associated with increased activity in the vmPFC (Fig. $2 B ;-6,57,0 ; p=0.05$; WBC; Table 3 ). Increasing dissent was associated with increased activity in classic error monitoring areas, namely the $\mathrm{dACC}(-1,8,51 ; p=0.012 \mathrm{SVC}$ and $p<0.05 \mathrm{WBC}$ ) and the intraparietal sulcus ( $p<0.05 \mathrm{WBC})$, a region frequently activated during the RDM task (Fig. $2 B$; Table $3)$. The latter finding suggest that the decision to choose an estimate different than the partner's estimate, results in an error or conflict signal (Fig. 2D). Furthermore, the dACC was more active

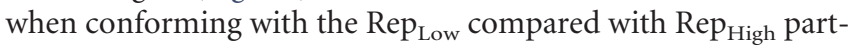
ner $(p<0.05$ WBC; Table 3$)$. This region overlapped with the conflict/error signal observed during dissent and with the peak regions found in a meta-analysis of neural activity using the term "error" conducted on 357 studies in Neurosynth (Fig. 2D). Finally, a $2\left(\operatorname{Rep}_{\mathrm{High} /} \operatorname{Rep}_{\text {Low }}\right) \times 2$ (Easy RDM/uncertain RDM conditions) ANOVA was used to examine the neural interaction between easy and uncertain RDM conditions and high and low reputation. This analysis showed activity in the social cognition network including the mPFC, temporoparietal junction (TPJ), and temporal pole in socially influenced perceptual decisionmaking $(p<0.05$ WBC; Table 4$)$.

Finally, we did not observe any behavioral difference between low reputation partner trials and no partner trials. Similarly, when we compared the neural activations between low reputation and no partner conditions, we found no neural differences (at $p<0.5$ FDR correction).

Partner reputation and threat-evoked anxiety

Being paired with $\mathrm{Rep}_{\text {Low }}$ compared with Rep $\mathrm{p}_{\text {High }}$ resulted in suggestively higher levels of subjective anxiety, assessed immediately
Table 4. Brain activation for interaction between reputation level and task difficulty (task period)

\begin{tabular}{lllllll}
\hline & & & \multicolumn{5}{c}{ Coordinates } \\
\cline { 6 - 8 } Brain region & Left/right & Cluster size & $t$ score & $x$ & $y$ & $z$ \\
\hline Medial frontal cortex & $\mathrm{R}$ & 1011 & 6.63 & 2 & 58 & -2 \\
Precuneus & $\mathrm{L}$ & 371 & 5.51 & -3 & -62 & 27 \\
TPJ & $\mathrm{L}$ & 235 & 5.46 & -47 & -60 & 28 \\
ACC & $\mathrm{R}$ & 184 & 4.96 & 3 & 37 & 18 \\
Insula & $\mathrm{R}$ & 150 & 4.85 & 42 & 36 & 33 \\
\hline All $p<0.05$, corrected for multiple comparisons (FDR). & & & & &
\end{tabular}

before the $4-6 \mathrm{~s}$ shock anticipation period $\left(t_{(21)}=-3.65, p=\right.$ 0.002; one-sample $t$ test; Fig. $3 A$ ). The Rep Low $_{\text {and Rep }}$ High partners performed equally well on average between levels of partner reputation. By design, partner performance was manipulated orthogonally such that some partners performed well at the task and others performed relatively poorly. Actual partner performance was defined as the sum total of error (difference between actual numerical motion coherence and partner's judgment) in the three perceptual judgments looped through for each stimulus. Actual performance modulated anxiety as much as reputation did: mean anxiety was significantly higher when the partner's performance was low compared with high $\left(t_{(21)}=-3.17, p=0.006\right.$; onesample $t$ test). There was no interaction between partner reputation and partner performance on anxiety.

To further investigate what caused participants' anxiety, we ran a multiple linear regression model with subjective anxiety ratings as the dependent variable. The variables included as predictors were the partner's implicit competence, reputation rating (i.e., 1 and 5 stars), partner's performance for the block, participant's performance for the block, and the overall level of social influence in the block (indexed by the total absolute difference between partner and participant numerical coherence judgments). Results of the model indicate that partner reputation, partner performance, and amount of social influence are each significantly associated of anxiety, whereas implicit partner competence and the participant's performance were not significantly associated with anxiety (Fig. 3B).

Next, we analyzed the neural correlates of how partner reputation affected subjective anxiety during the shock anticipation period (trials with actual shocks were removed from the analysis; Table 5). Being paired with a $\operatorname{Rep}_{\text {Low }}$ compared with a Rep $\mathrm{P}_{\mathrm{High}}$ partner increased activity in anticipatory pain pathways, namely the dACC and bilateral posterior insula (pINS; $p<0.05 \mathrm{WBC}$; Fig. $3 C, D)$. We also tested whether there were regions selectively more or less active during anticipation as a function of partner actual performance, but no regions survived multiple-comparisons correction. Finally, we conducted a psychophysiological (PPI) analysis which showed the connectivity from the pINS to dACC $(p<0.05$ WBC; Fig. 3E; Table 6), consistent with earlier findings about pain pathways (Wilcox et al., 2015).

\section{Discussion}

How others influence our behavior has been the target of social science research for decades. Based on previous research and theory, we hypothesized that Rep High $_{\text {partners would create greater }}$ levels of social influence on perceptual judgments and lower anxiety toward a potential threat. We found that a partner's reputation did have an impact on the perception of RDM perceptual estimates, especially as stimulus uncertainty increased. We also found that a partner's reputation affected subjective and neural measures of anxiety, and levels of a partner's reputation were 


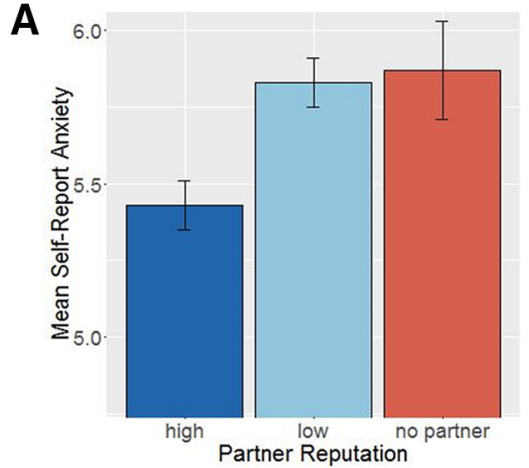

C

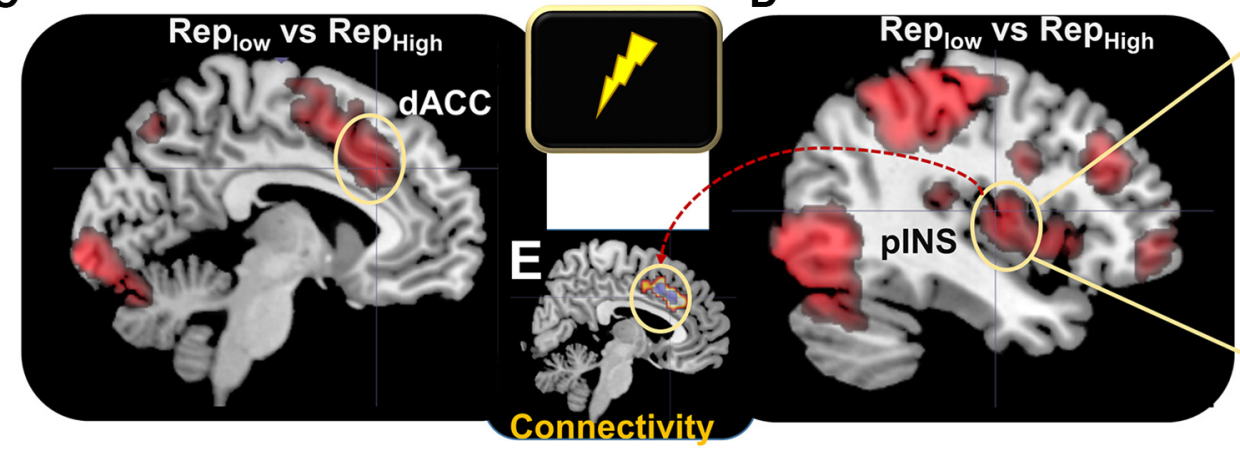

B

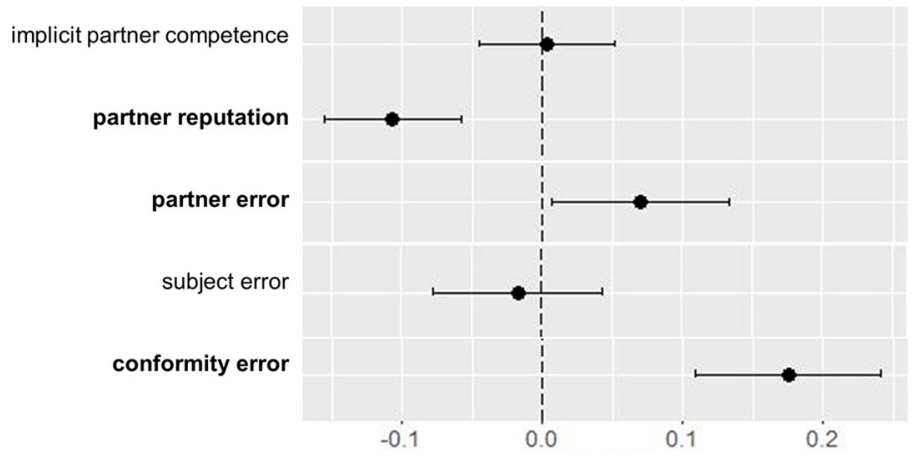

Standardized beta coefficient $95 \%$ confidence intervals

Figure 3. $\boldsymbol{A}$, Effect of partner reputation on self-report anxiety. $\boldsymbol{B}$, Regression coefficients comparing partner reputation to other variables that potentially affect anxiety ratings. $\boldsymbol{C}$, fMRI activity during anxious anticipation of potential shock for Rep Low $_{\text {compared with Rep }}$ High. Signal change reflects activity in the (C) dACC and (D) pINS. Betas show the differences in activity for Rep Low $_{\text {and }}$ Rep $_{\text {High }}$ for both the ambiguous (uncertain) and easy RDM conditions, using an independent ROI taken from Tedeschi et al. (2015). $\boldsymbol{E}$, connectivity between the pINS (seed) and dACC.

Table 5. Brain activation for contrast [low reputation- high reputation (shock anticipation period)]

\begin{tabular}{|c|c|c|c|c|c|c|}
\hline \multirow[b]{2}{*}{ Brain region } & \multirow[b]{2}{*}{ Left/right } & \multirow[b]{2}{*}{ Cluster size } & \multirow[b]{2}{*}{$t$ score } & \multicolumn{3}{|c|}{ Coordinates } \\
\hline & & & & $x$ & $y$ & $z$ \\
\hline \multicolumn{7}{|c|}{ Low-high reputation (anticipation) } \\
\hline Middle occipital gyrus & $\mathrm{L}$ & 822 & 8.88 & -21 & -96 & 6 \\
\hline Calcarine & $\mathrm{R}$ & 761 & 8.38 & 12 & -99 & 0 \\
\hline Insula & $\mathrm{R}$ & 235 & 7.46 & 44 & 14 & 3 \\
\hline Precentral gyrus & $\mathrm{L}$ & 625 & 6.84 & -42 & -18 & 57 \\
\hline Middle frontal cortex & $\mathrm{R}$ & 146 & 6.25 & 42 & 36 & 33 \\
\hline
\end{tabular}

All $p<0.05$, corrected for multiple comparisons (FDR).

Table 6. Brain activation for PPI analysis (pINS seed)

\begin{tabular}{|c|c|c|c|c|c|c|}
\hline \multirow[b]{2}{*}{ Brain region } & \multirow[b]{2}{*}{ Left/right } & \multirow[b]{2}{*}{ Cluster size } & \multirow[b]{2}{*}{ tscore } & \multicolumn{3}{|c|}{ Coordinates } \\
\hline & & & & $x$ & $y$ & $z$ \\
\hline Insula & L & 154 & 3.90 & -39 & 12 & 0 \\
\hline Middle frontal gyrus & L & 70 & 3.86 & -45 & 21 & 33 \\
\hline Precentral gyrus & $\mathrm{R}$ & 317 & 3.71 & 54 & 0 & 18 \\
\hline $\mathrm{dACC}$ & L & 227 & 3.68 & -3 & 15 & 39 \\
\hline
\end{tabular}

All $p<0.05$, corrected for multiple comparisons (FDR).

more predictive of anxiety than any other measure. Our observations build on social influence frameworks by linking the neural representations of perceived reputation with decision-making and emotion.

The bilateral amygdala was significantly more active when the subjects were shown the face and reputation (i.e., number of

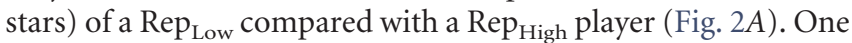
explanation for this result is that pairing with subject with a Re$\mathrm{p}_{\text {Low }}$ partner resulted in a threat or saliency signal, potentially associated with the increasing likelihood of diminished perfor- mance, and hence, the increased chance of an electric shock. Research shows that the amygdala is evoked during explicit judgements of untrustworthiness (Adolphs et al., 1998; Winston et al., 2002), yet these studies do not link such judgments to behavioral outcomes. An alternative explanation might be that the amygdala plays a role in invigorating the subject to work harder at the task, although if this estimation true, one might expect to see activity in the other dopamine enriched areas, such as the ventral striatum (Pessiglione et al., 2007) which was not observed, making this conclusion less viable. Thus, the amygdala activity might be a signal for threat and behavioral avoidance (Adolphs, 2010; Mobbs et al., 2013) associated with the increased potential for harm during performance outcome.

Parametric neural activity in the dACC increased as partnerparticipant perceptual conflict increased, whereas activity in a vmPFC region increased as the amount of social influence, or perceptual similarity, increased. In line with our findings, this dACC region associated with dissent or social conflict, has previously been linked to conflict or error monitoring, and the more ventral $\mathrm{mPFC}$ region here associated with conformity may contribute to integrating socially relevant reward signals (Mobbs et al., 2015). Indeed, the most consistent findings in fMRI studies of conformity show the dACC to be involved in anticonformity or dissent (Berns et al., 2005; Campbell-Meiklejohn et al., 2010; Izuma and Adolphs, 2013). Recent controversy has, however, occluded the role of the dACC, including its role in motivating effortful behavior or expected value of control (Shenhav et al., 2013). Without directly comparing these theories empirically, it is difficult to state the psychological role of the dACC, yet each of these theories provides viable explanations for our data. It is also important to note that we also found increased activity in the IPS, 
a region involved in perceptual judgments similar to those used in this study (Newsome et al., 1989; Raymond, 2000; Braddick et al., 2001), where it is possible that this reflects greater recruitment of motion processing regions as the participant's judgment deviated from the partner's, or equivalently, less recruitment of motion processing resources when conforming more closely.

Another key finding of this study was that the participants' perceptions of uncertain stimuli were influenced more by Rep $\mathrm{p}_{\text {High }}$ than $\operatorname{Rep}_{\text {Low }}$ partners. This reputation effect interacted with task difficulty such that participants were not influenced at significantly different rates when the task was easy, yet as the task difficulty (i.e., stimulus uncertainty) increased, so did the difference in levels of influence between $\mathrm{Rep}_{\mathrm{High}}$ and Rep $\mathrm{p}_{\mathrm{Low}}$ partners. This finding supports informational social influence theory, or in

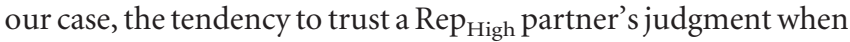
the RDM task was difficult to estimate (Wooten and Reed, 1998). The neural data showed that when the subjects conformed with

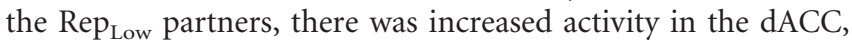
which overlapped with overall increased dissent and a metaanalysis of studies examining the neural basis of error. Although this suggests an alternative view of the dACC to the parametric analysis discussed above, it does fit with the notion that this region is involved in error monitoring or increased perceptual effort. The later theory seems unlikely, however, as activity in the dACC did not correlate with performance $(p=0.22, r=0.19)$. Finally, we conducted an interaction between $\operatorname{Rep}_{\text {High }} / \operatorname{Rep}_{\text {Low }} \times$ Easy/Uncertain RDM conditions, which revealed a role for the social cognition network, including the TPJ, mPFC, temporal pole, and dACC (Stanley and Adolphs, 2013), suggesting that these regions are involved in integrating social information with perceptual judgments.

Chief among our hypotheses is the notion that a partner's reputation can shape subjective ratings of anxiety. Previous work has demonstrated that when one's risk for a shock depends on the task performance of an unfamiliar person, the level of perceived competence in their appearance alone, modulates subjective, and neural measures of anxiety (Tedeschi et al., 2015). In the present task, having a Rep High $_{\text {partner alone decreased anxiety ratings }}$ toward a possible shock compared with having a Rep Low $_{\text {partner }}$ or no partner at all. We also see this decrease in brain regions that respond to the anticipation of shocks including the pINS, somatosensory cortex, and AACC where there was less activity during the anticipation period for the $\mathrm{Rep}_{\mathrm{High}}$ compared with the Rep $_{\text {Low }}$ partner. The pINS region appears to largely overlap with the regions observed in previous pain studies (Tedeschi et al., 2015), perhaps indicating this region, known to be involved in interoception and pain processing (Craig, 2003a,b), is sensitive to these two different cues of another's reputation and, accordingly, one's risk of harm.

There has been a growing body of literature demonstrating a series of social contexts that influence decision-making and cognition. Studies have shown that information from experts can enhance subsequent memory effects (Klucharev et al., 2008) and decision-making processes (Boorman et al., 2013). Further, the opinions of others and group membership can also modulate choices and value judgments (Izuma et al., 2008; CampbellMeiklejohn et al., 2010). These contextual effects were associated with activities in the ventral striatum, STS, and prefrontal regions, which process value during social interaction (Mobbs et al., 2015). Reputation, however, is significant because it shows that without experience, one can be led to trust others opinions. Reputation is likely one of many cues that drive us to conform to others (e.g., expectations, confidence, metacognitive abilities, and trust). Therefore, a future goal is to understand the variety of social variables that lead people to conform to other opinions.

These findings have implications across all fields in which people make judgments in a collectively way (e.g., in teamwork), or are influenced by what others think and say. In politics, peer judgments can influence voting behavior based on limited data. In organizations, committees often make decisions about hiring and risky investments aggregating judgments of people who know a lot or a little. In economics and finance "herding" can be rational; helping less-informed investors rely on superior knowledge of others. In medicine, patient-practitioner interactions require patients to weigh how much their doctor knows, or to compare initial and second opinions to avoid harmful results. In law enforcement and combat, opinions are shared during lifeand-death decisions. Game theory goes even further, by considering the incentives of experts to misrepresent how much they know, and how well consumers of expert opinion adjust for these incentives (Crawford and Sobel, 1982). Future research, therefore, may be able to elucidate how the effects of reputation described here, reflect more general ways humans integrate cues about peer knowledge in the social environment and how they bias cognition and emotion.

\section{References}

Adolphs R (2010) What does the amygdala contribute to social cognition? Ann N Y Acad Sci 1191:42-61. CrossRef Medline

Adolphs R, Tranel D, Damasio AR (1998) The human amygdala in social judgment. Nature 393:470-474. CrossRef Medline

Asch SE (1956) Studies of independence and conformity: I. A minority of one against a unanimous majority. Psychol Monogr Gen Appl 70:1-70. CrossRef

Beckes L, Coan JA (2011) Social baseline theory: the role of social proximity in emotion and economy of action. Soc Personal Psychol Compass 5:976988. CrossRef

Berns GS, Chappelow J, Zink CF, Pagnoni G, Martin-Skurski ME, Richards J (2005) Neurobiological correlates of social conformity and independence during mental rotation. Biol Psychiatry 58:245-253. CrossRef Medline

Boorman ED, O’Doherty JP, Adolphs R, Rangel A (2013) The behavioral and neural mechanisms underlying the tracking of expertise. Neuron 80:1558-1571. CrossRef Medline

Braddick OJ, O’Brien JM, Wattam-Bell J, Atkinson J, Hartley T, Turner R (2001) Brain areas sensitive to coherent visual motion. Perception 30:6172. CrossRef Medline

Craig AD (2003a) Interoception: the sense of the physiological condition of the body. Curr Opin Neurobiol 13:500-505. CrossRef Medline

Craig AD (2003b) A new view of pain as a homeostatic emotion. Trends Neurosci 26:303-307. CrossRef Medline

Campbell-Meiklejohn DK, Bach DR, Roepstorff A, Dolan RJ, Frith CD (2010) How the opinion of others affects our valuation of objects. Curr Biol 20:1165-1170. CrossRef Medline

Cialdini R (1993) The psychology of influence. New York: William Morrow.

Cialdini RB, Goldstein NJ (2004) Social influence: compliance and conformity. Annu Rev Psychol 55:591-621. CrossRef Medline

Coan JA, Schaefer HS, Davidson RJ (2006) Lending a hand: social regulation of the neural response to threat. Psychol Sci 17:1032-1039. CrossRef Medline

Crawford VP, Sobel J (1982) Strategic information transmission. Econometrica 50:1431-1451. CrossRef

Genovese CR, Lazar NA, Nichols T (2002) Thresholding of statistical maps in functional neuroimaging using the false discovery rate. Neuroimage 15:870-878. CrossRef Medline

Izuma K, Adolphs R (2013) Social manipulation of preference in the human brain. Neuron 78:563-573. CrossRef Medline

Izuma K, Saito DN, Sadato N (2008) Processing of social and monetary rewards in the human striatum. Neuron 58:284-294. CrossRef Medline

Kikusui T, Winslow JT, Mori Y (2006) Social buffering: relief from stress 
and anxiety. Philos Trans R Soc Lond B Biol Sci 361:2215-2228. CrossRef Medline

Klucharev V, Smidts A, Fernández G (2008) Brain mechanisms of persuasion: how "expert power" modulates memory and attitudes. Soc Cogn Affect Neurosci 3:353-366. CrossRef Medline

Krahé C, Springer A, Weinman JA, Fotopoulou A (2013) The social modulation of pain: others as predictive signals of salience: a systematic review. Front Hum Neurosci 7:386. CrossRef Medline

Milgram S (1964) Group pressure and action against a person. J Abnorm Psychol 69:137-143. CrossRef Medline

Mineka S, Cook M (1986) Immunization against the observational conditioning of snake fear in rhesus monkeys. J Abnorm Psychol 95:307-318. CrossRef Medline

Mobbs D, Hassabis D, Yu R, Chu C, Rushworth M, Boorman E, Dalgleish T (2013) Foraging under competition: the neural basis of input-matching in humans. J Neurosci 33:9866-9872. CrossRef Medline

Mobbs D, Hagan CC, Yu R, Takahashi H, FeldmanHall O, Calder AJ, Dalgleish T (2015) Reflected glory and failure: the role of the medial prefrontal cortex and ventral striatum in self vs other relevance during advice-giving outcomes. Soc Cogn Affect Neurosci 10:1323-1328. CrossRef Medline

Newsome WT, Britten KH, Movshon JA (1989) Neuronal correlates of a perceptual decision. Nature 341:52-54. CrossRef Medline

Pessiglione M, Schmidt L, Draganski B, Kalisch R, Lau H, Dolan RJ, Frith CD (2007) How the brain translates money into force: a neuroimaging study of subliminal motivation. Science 316:904-906. CrossRef Medline

Petrovic P, Dietrich T, Fransson P, Andersson J, Carlsson K, Ingvar M (2005) Placebo in emotional processing: induced expectations of anxiety relief activate a generalized modulatory network. Neuron 46:957-969. CrossRef Medline
Raymond JE (2000) Attentional modulation of visual motion perception. Trends Cogn Sci 4:42-50. CrossRef Medline

Schachter S (1951) Deviation, rejection, and communication. J Abnorm Psychol 46:190-207. CrossRef Medline

Shenhav A, Botvinick MM, Cohen JD (2013) The expected value of control: an integrative theory of anterior cingulate cortex function. Neuron 79: 217-240. CrossRef Medline

Sherif M (1935) A study of some social factors in perception. New York. Columbia University.

Stanley DA, Adolphs R (2013) Toward a neural basis for social behavior. Neuron 80:816-826. CrossRef Medline

Sterzer P, Frith C, Petrovic P (2008) Believing is seeing: expectations alter visual awareness. Curr Biol 18:R697-R698. CrossRef Medline

Tedeschi E, Weber J, Prévost C, Mischel W, Mobbs D (2015) Inferences of others' competence reduces anticipation of pain when under threat. J Cogn Neurosci 27:2071-2078. CrossRef Medline

Todorov A, Mandisodza AN, Goren A, Hall CC (2005) Inferences of competence from faces predict election outcomes. Science 308:1623-1626. CrossRef Medline

Wilcox CE, Mayer AR, Teshiba TM, Ling J, Smith BW, Wilcox GL, Mullins PG (2015) The subjective experience of pain: an FMRI study of perceptrelated models and functional connectivity. Pain Med 16:2121-2133. CrossRef Medline

Winston JS, Strange BA, O’Doherty J, Dolan RJ (2002) Automatic and intentional brain responses during evaluation of trustworthiness of faces. Nat Neurosci 5:277-283. CrossRef Medline

Wooten DB, Reed A (1998) Informational influence and the ambiguity of product experience: order effects on the weighting of evidence. J Consum Psychol 7:79-99. CrossRef 